\title{
TENTATIVA DE SUICÍDIO COM O USO DE MEDICAMENTOS REGISTRADOS PELO CIT-GO NOS ANOS DE 2003 E 2004
}

\section{Suicide attempt with drug self administration recorded on the Goias state center of toxicological information over the years 2003 and 2004}

\author{
Diogo P. Rios ${ }^{1}$, Fernando M. Bastos ${ }^{1}$, Luiz C. da Cunha ${ }^{2}$, Marize C. Valadares ${ }^{2}$ \\ ${ }^{1}$ Graduandos de Farmácia da Faculdade de Farmácia, Universidade Federal de Goiás \\ ${ }^{2}$ Professores de Toxicologia da Faculdade de Farmácia da Universidade Federal de Goiás e coordenadores do \\ Núcleo de Estudos e Pesquisas Tóxico-Farmacológicas (NEPET-UFG). \\ Autor para correspondência: Iccunha@farmacia.ufg.br.
}

*Autor para correspondência: e-mail: mcvaladares@farmacia.ufg.br

Recebido em 22/06/2005 - aceito em 04/07/2005

\begin{abstract}
RESUMO: A ingestão acidental ou proposital de agentes potencialmente tóxicos é responsável pela maioria das intoxicações graves. Considerando todo o universo de intoxicações, a partir das mais leves às graves, as mais comuns são provocadas por medicamentos, principalmente os ansiolíticos, antidepressivos, analgésicos e anticonvulsivantes. A tentativa de suicídio é bastante comum, embora não seja diagnosticada como tal por médicos, por falta de vivência constante com os distúrbios psiquiátricos. O trabalho avaliou e comparou os casos de tentativa de auto-extermínio, causados pela ingestão de medicamentos, registrados pelo Centro de Informações Toxicológicas do Estado de Goiás (CIT-GO) nos anos de 2003 e 2004. Foram notificados 597 casos provocados por medicamentos no insterstício. No período de 2003 houve 237 casos, enquanto que no ano de 2004, 360 casos, 50\% a mais de notificações. Os psicofármacos, os analgésicos e os antinflamatórios nãoesteroidais foram responsáveis por mais de $60 \%$ de todos os casos de tentativas de suicídio. Cerca de $80 \%$ ocorreu com o sexo feminino, principalmente na faixa etária de 21 a 30 anos, com baixo índice de letalidade $(0,84$ $-2,49 \%)$.
\end{abstract}

PALAVRAS-CHAVE: fármacos, produtos farmacêuticos, suicídio.

\begin{abstract}
Accidental ou proposital administratrion of potentially toxic agents is related to severe intoxications. Considering all classes of intoxications (minor to severe) the majority are caused by drugs, like as antidepressant, analgesic, anti-anxiety and anticonvulsant agents. The suicide attempt is very common, instead of clinical or forensic misdiagnostic of such psychiatric disturbs. In this work we evaluated and compared suicide attempt, caused by medicine ingestion, recorded on Center of Toxicological Information of the Goias State (CITGO) over the years 2003 and 2004. In such period, 597 cases of poisoning were related to medicines. In the period of 2003, 237 cases had been notified, while that in the year of 2004 had been notified 360 cases, 1.5 times more than the previous year. The psychiatric drugs, analgesics and non-steroidal antinflamatory drugs (NSAIDS) had been responsible for more than $60 \%$ of all the cases of suicide attempts. About $80 \%$ of the medecine suicide attempt cases it occurred in the feminine sex, mainly in the age range of 21 the 30 years, with low index of lethality $(0,84-2.49 \%)$.
\end{abstract}

KEY WORDS: drugs, pharmaceutical products, suicide. 


\section{INTRODUÇÃO}

A Toxicologia, tradicionalmene, é conceituada como sendo a ciência que estuda os efeitos adversos e tóxicos provocados pelas substâncias sobre os organismos vivos. Este era o conhecimento aceito no início do século XX. Atualmente, esta definição deve ser ampliada: é uma ciência multidisciplinar que, além de estudar os efeitos adversos causados por agentes químicos no homem e no meio ambiente, estuda também as propriedades físico-químicas de cada substância e avalia a segurança de seu uso (MÍDIO, 1992; OGA, 2004).

Existem relatos de milhares de anos que mostra que o homem utilizava venenos animais e extraídos de plantas para a caça, a guerra e até mesmo para assassinatos. Ao longo da história, há vários relatos de suicídios, onde a vítima utilizou "conhecimentos toxicológicos" para obter êxitos em seu intento.

As intoxicações são causas freqüentes de procura de atendimento médico em serviços de urgência e emergência em todo o mundo. A American Association of Poison Control Centres Toxic Expossure Surveillance System (EUA) registrou, em 1994, quase dois milhões de exposições a agentes potencialmente tóxicos (drogas e toxinas), mas estima-se que o número real seja próximo de quatro milhões de casos, uma vez que muitas intoxicações não são notificadas (AMDUR et al, 1991).

Apesar deste grande número de casos registrados, a taxa de letalidade é aparentemente baixa. Isto não significa que as intoxicações sejam, sempre, casos benignos. Alguns fatores contribuem para que se tenha esta falsa idéia. Neste levantamento não foram computados os óbitos ocorridos antes do diagnóstico ou mesmo antes dos pacientes receberem cuidados médicos. Além disso, a intervenção médica precoce e eficaz em situações de ameaça à vida, como intoxicação por cianeto, hipoglicemiantes, anticolinesterásicos, barbitúricos, antidepressivos e monóxido de carbono, contribuem para diminuir a taxa de letalidade (ELLENHORN, 1997; GOSSEL \& BRICKER, 1994; HARDMAN \& LIMBIRD, 2003).

Considerando todo o universo de intoxicações, das leves às graves, as mais comuns são provocadas por medicamentos, principalmente os ansiolíticos, antidepressivos, analgésicos e anticonvulsivantes. Apesar da maioria dos óbitos estar relacionada a exposição a esses agentes, a resposta a exposição a uma substância é individual e o médico assistente pode observar manifestações variadas, em pacientes diferentes, mas expostos a mesma substância. A resposta da vítima pode variar desde pequena alteração na região da pele que entrou em contato com o agente até reações sistêmicas graves (e.g.: choque anafilático) em outra vítima (SINITOX).

Várias circunstâncias podem levar a um quadro de intoxicação: Exposição profissional ou acidental, abuso, tentativa de suicídio e homicídios. As intoxicações decorrentes de tentativa de auto-extermínio tendem a ser mais graves que nas outras circunstâncias. Isto pode ser explicado pela escolha de tóxico mais potente, uma exposição mais prolongada ou ingestão de maior quantidade do veneno, além de retardo, provocado pela própria vítima, no atendimento inicial (KAPUR et al, 2005).

A ingestão acidental ou proposital de agentes potencialmente tóxicos é responsável pela maioria das intoxicações graves. Estas podem ser revertidas com alguns procedimentos se realizados a tempo. A descontaminação gástrica é um procedimento simples, que não requer nenhum equipamento sofisticado e, quando realizados em tempo hábil, diminui o potencial tóxico da substância ingerida. O momento ideal varia de acordo com o agente tóxico. De modo geral, deve ser feita até no máximo duas horas após a ingestão, com exceção de substâncias que retardam o esvaziamento gástrico, como barbitúricos, anticolinérgicos, antidepressivos tricíclicos. A descontaminação gástrica pode ser feita através de indução de vômitos ou da lavagem gástrica (ANDRADE FILHO et al, 2001).

A indução de vômitos, em estudos com voluntários, mostrou recuperação de $21 \%$ a $38 \%$ do agente tóxico, se realizada até uma hora após a ingestão. Poucos pacientes intoxicados chegam a um serviço de urgência com este tempo de evolução, portanto, tem sido cada vez menos indicada. Ela não deve ser indicada em pacientes que ingeriram substâncias cáusticas, derivados de petróleo, depressores do SNC e agentes convulsivantes. Também está contra-indicado no último trimestre de gravidez e em pacientes com convulsão, devido ao risco de precipitar aborto ou levar a aspiração de conteúdo gástrico. Existem vários agentes que podem ser usados para provocar vômitos: apomorfina, xarope de ipeca e detergente de $\mathrm{pH}$ neutro. Estudos realizados em voluntários que se submeteram ao procedimento de lavagem gástrica, demonstraram recuperação de $32 \%$ do agente tóxico se for realizada até uma hora após a ingestão (ELLENHORN, 1997; ANDRADE FILHO et al, 2001).

Outro método de descontaminação é através do uso de substâncias adsorventes, que têm a capacidade de se ligarem ao agente tóxico formando um composto estável que não é absorvido pelo trato gastrointestinal e é eliminado pelas fezes. O carvão ativado é usado por via oral ou por sonda naso ou orogástrica. Estudos feitos em voluntários mostraram que a eficácia máxima do carvão ativado é verificada se for administrado até cerca de uma hora após a ingestão do agente tóxico. Além da adsorção, o carvão ativado tem a propriedade de fazer uma "diálise gastrointestinal" para algumas substâncias, como fenobarbital, aminofilina, fenitoína, e salicilatos. Ele também interrompe a recirculação êntero-hepática nas intoxicações por antidepressivos tricíclicos, hormônio tireoidiano e carbamazepina (ANVISA, 2004). 
Em pacientes intoxicados que já não se beneficiam de medidas de descontaminação, pode-se utilizar métodos que aumentam a excreção dos agentes tóxicos, como diurese forçada, alcalinização de urina, hemodiálise e hemoperfusão em filtro de carvão (WHO, 2005).

A grande maioria dos pacientes intoxicados são atendidos, inicialmente, em unidades de urgência e emergência, geralmente em situação de estresse para o paciente, familiares, acompanhantes (policiais, socorristas) e para a equipe médica. Se o paciente está em condições de falar, é dele que se deve colher os dados iniciais, que depois serão checados com os acompanhantes, principalmente em tentativa de autoextermínio, pois a história pode ser deliberadamente falseada pelo paciente (ANDRADE FILHO et al, 2001).

Em cerca de $50 \%$ de tentativa de auto-extermínio, é falsa a informação fornecida pela vítima sobre o nome tóxico e a quantidade ingerida. Caso o paciente esteja comatoso, a história deve ser colhida com os acompanhantes. Se esta fonte for insuficiente, localizar o telefone da casa ou do trabalho do paciente, para que se consiga detalhes sobre seus hábitos e do que ocorreu antes da admissão no hospital (KAPUR et al, 2005).

No levantamento da história deve ser dada atenção especial para a profissão do paciente, qual ambiente de trabalho, atividades domésticas, presença de medicamentos, pesticidas e produtos de limpeza, hábitos (uso de drogas), passado de atopia, doenças prévias (cardíaca, pulmonar, psiquiátrica, neurológica, hepática, renal ou hematológica), passado ou história familiar de tentativa de suicídio. Estes dados podem fornecer informações importantes sobre as possíveis intoxicações e orientar os exames complementares específicos e o tratamento (ELLENHORN, 1997; KAPUR et al, 2005).

Outro ponto que deve ser investigado é se no local onde o paciente foi encontrado havia algum resto de alimento, cartelas ou frascos de medicamentos, garrafas de bebida alcoólica, caixas ou frascos de pesticidas, "tralha" ou "parafernália" para uso de drogas (cachimbos, canudos, etc), que despertarão o examinador para a possibilidade da intoxicação (ANDRADE FILHO et.al, 2001).

A tentativa de suicídio é bastante comum, embora não seja diagnosticada como tal por médicos, por falta de vivência constante com os distúrbios psiquiátricos (PONTES, 1998a).

Analisando os inúmeros suicídios e tentativas nestes últimos anos, e considerando reflexões filosóficas desde Platão, chegamos à conclusão de a morte acontece de forma "acidental", pois não existe no mundo alguém que queira realmente morrer. O que o indivíduo não admite na sua onipotência, é ficar condenado a uma doença incurável, a uma calúnia, a uma traição, a uma perda financeira importante. A pessoa quer é escapar de tudo isso, ter uma vida melhor e o suicídio surge como uma tentativa mágica para a resolução dos problemas (PONTES, 1998b; KAPUR et al, 2005).

Para STENGEL (1964), a tentativa de suicídio significa um apelo; em outras palavras, tal gesto exerce "uma função de apelo". Significando, em nível terapêutico, que quando a comunicação é bem sucedida, o apelo compreendido, o ato não se concretiza; já um fracasso na comunicação geralmente determina o sucesso no ato. Do ponto de vista psiquiátrico, constatamos que o estado depressivo lidera as tentativas de suicídio, pela baixa auto-estima, sentimento de pouca valia, niilismo, sintomas comuns a esse quadro.

O conceito de depressão pode não ser bem compreendido por leigos ou até mesmo por médicos de outras áreas, daí ser oportuno investigar melhor os hábitos de um paciente suspeito de estar num grupo de risco. É importante saber, por exemplo, se ele vem bebendo em demasia, se vem tomando tranqüilizantes, dirigindo periculosamente, sofrendo acidentes, com distúrbios do sono. Tais comportamentos podem ser condutas autodestrutivas, com tendência a uma progressão. Sendo assim, nestes casos uma boa anamnese deve ser realizada (PONTES, 1998a).

Segundo BORTOLETTO E BOCHNER, 1999, no período de 1993 a 1996, foram registrados no Brasil, pelo Sistema Nacional de Informações Tóxico-Farmacológicas (SINITOX), 217.512 casos de intoxicação humana, com um total de 1.483 óbitos. Nesse período, os medicamentos se destacaram entre os agentes tóxicos, contribuindo com $27 \%$ dos casos registrados pela Rede de Centros de Controle de Intoxicações e ocupando o primeiro lugar nas estatísticas relativas a esses eventos. No conjunto dos 13 agentes tóxicos considerados pelo SINITOX, os medicamentos responderam por $62 \%$ das tentativas de suicídio registradas no período. Neste, a participação do sexo feminino nos casos registrados de intoxicação por medicamentos foi bastante expressiva: dos 57.748 casos, $63 \%$ eram mulheres.

\section{OBJETIVOS}

Avaliar e comparar os casos de tentativa de auto-extermínio, causados pela ingestão de medicamentos, registrados pelo Centro de Informações Toxicológicas do Estado de Goiás (CIT-GO) nos anos de 2003 e 2004. 


\section{METODOLOGIA}

Para elaboração deste trabalho, a coleta de dados foi feita através de cinco visitas no CIT-GO, entre os meses de maio e abril de 2005. Os dados foram colhidos através de fichas de notificação referentes a intoxicação por medicamentos nos anos de 2003 e 2004 registradas pelo mesmo no Estado de Goiás. Entre as diversas causas de intoxicação, foram selecionadas aquelas referentes a tentativa de suicídio, totalizando 597 fichas, sendo 237 do ano de 2003 e 360 do ano de 2004 e separadas segundo: sexo, faixa etária, evolução dos pacientes, número de medicamentos ingeridos e nome dos fármacos/medicamentos (nome genérico da Denominação Comum Brasileira). Os dados foram analisados por meio de estatística descritiva.

Os medicamentos foram agrupados nas seguintes classes: analgésicos (analgésicos, antipiréticos e antiinflamatórios), anorexígenos, antibióticos, antidepressivo, anticonvulsivante, benzodiazepínicos, fármacos cardiovasculares, e outros. Foram agrupadas em "outros" todas as classes de medicamentos que tiveram incidência menor que $3 \%$ no total de cada ano.

No ano de 2003 as classes consideradas como "outros" foram: antipsicóticos, antiemético, corticosteróides, espasmolítico, anticoncepcionais, sulfonamidas, anti-hanseníase, antifúngico, antiprotozóarios, diuréticos, fitoterápico, anti-asmáticos, anti-úlceras, vitaminas, anti-histâminicos, antianêmico, antiparkinsonianos, hipoglicemiante orais e antibióticos. Em 2004 as classes consideradas como "outros" foram: antibióticos antipsicóticos, antiemético, corticosteróides, espasmolítico, anticoncepcionais, sulfonamidas, anti-hanseníase, antifúngico, antiprotozóarios, diuréticos, fitoterápicos, anti-asmáticos, anti-úlceras, vitaminas, anti-histamínicos, anti-helminticos, e "boa noite cinderela" (e.g.: benzodiazepínico, gama-hidroxibutirato [GHB]) (KOROLKOVAS et al, 2000; SILVA, 2002; HARDMAN \& LIMBIRD et al, 2003).

\section{RESULTADOS E DISCUSSÃO}

Foram notificados 597 casos de intoxicação provocadas por medicamentos, em tentativas de suicídio, em 2003 e 2004. No período de 2003 foram notificados 237 casos, enquanto que no ano de 2004 foram notificados 360 casos. No período de 2004 ocorreram, aproximadamente, 50\% a mais de notificações.

O sexo feminino predominou nos casos de intoxicação, sendo a sua participação superior a $80 \%$ nos dois anos (Gráfico 1), corroborando com levantamentos semelhantes (KAPUR et al, 2005; SINITOX). Na nossa cultura, a mulher tenta dez vezes mais o suicídio do que o homem, embora ela consiga atingir a letalidade três vezes menos do que ele. Tal dado nos leva a ratificar a idéia de que uma tentativa de suicídio é um pedido de ajuda, é uma forma de comunicação, a qual a mulher demonstra melhor habilidade do que o homem, pois atinge os objetivos sem necessariamente prescindir da vida (PONTES, 1998a; PONTES, 1998b; KAPUR et al, 2005).

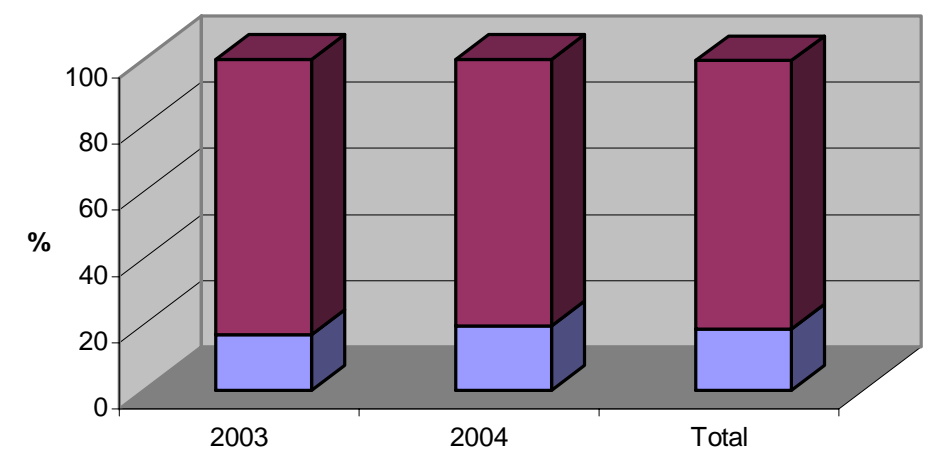

口Feminino

口Masculino

Gráfico 1: Distribuição por sexo dos pacientes intoxicados por medicamentos em tentativa de suicídio em 2003 e 2004 notificados pelo CIT-GO. 


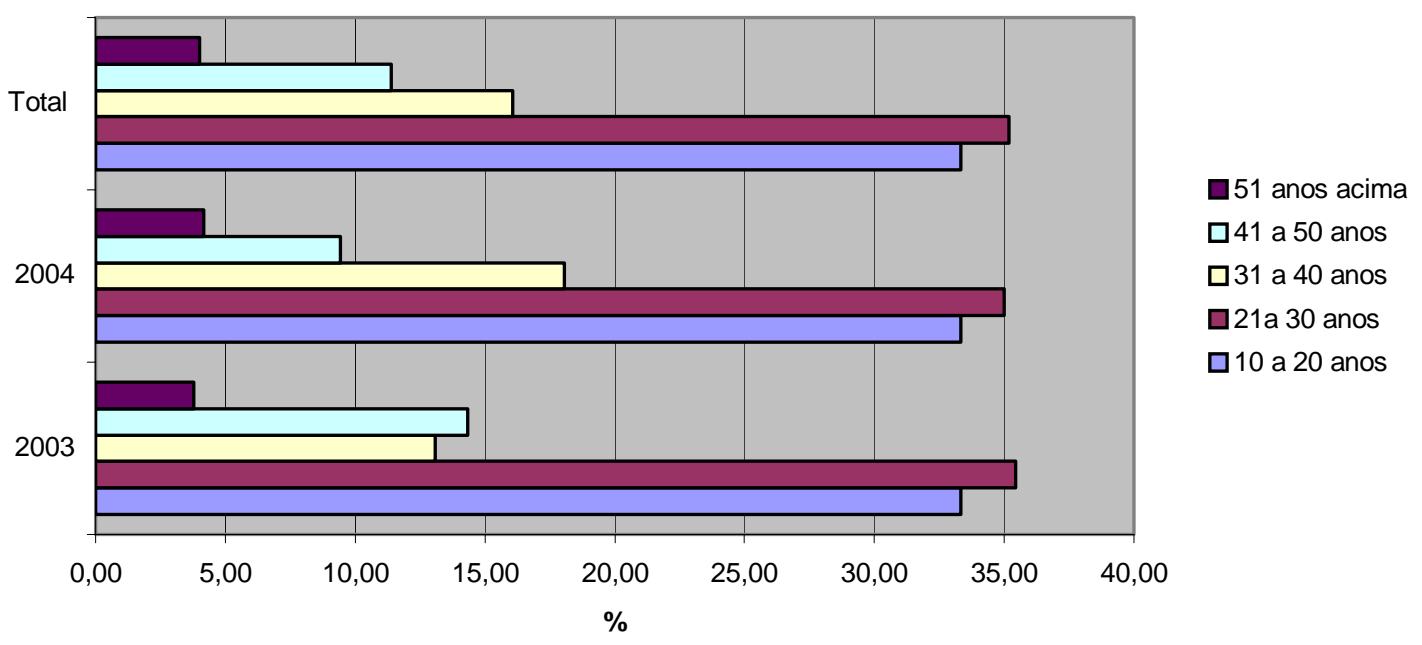

Gráfico 2: Distribuição por faixa etária dos pacientes intoxicados por ingestão de medicamentos em tentativa de suicídio notificados pelo CIT-GO em 2003 e 2004.

Quase $70 \%$ das tentativas de suicídio se concentram na faixa etária dos 10 a 30 anos (68,51\%), sendo faixa etária de 21 a 30 anos a predominante, apresentando aproximadamente $35 \%$ dos casos. A faixa etária de 31 a 40 anos contribui com $16 \%$ dos casos, enquanto a faixa etária de 41 a 50 anos apresenta $11 \%$ dos casos, a faixa etária acima de 51 anos contribui com apenas $4 \%$ dos casos (Gráfico 2).

A Organização Mundial de Saúde identifica o suicídio como uma das três principais causas de morte entre adolescentes e jovens adultos, e o problema se torna ainda maior considerando o número de jovens que possuem ideais suicidas e os casos em que as tentativas não evoluíram para óbito (WHO, 2001).

Raramente as crianças tentam o suicídio, pelo menos em nível consciente. Adotam mais atos de rebeldia ou de doenças recorrentes e, às vezes, acidentes, como a quebra de um braço ou de outros membros. Mas existem fases da vida em que devemos concentrar uma atenção maior, como o final da adolescência, onde prevalecem condutas periculosas, impulsivas. Porém, no nosso meio é comum observar atitudes francamente autodestrutivas em indivíduos de meia idade obrigando, às vezes, precisar se existe, ou não um processo de senilidade precoce debutante (PONTES, 1998a). Quando o diagnóstico é positivo, as tentativas são mais graves, como se elas refletissem o "caos" psíquico em que o paciente se encontra. Nesse caso, a família também deve ser trabalhada, pois envolve nesta situação a expectativa que os membros fazem em relação ao indivíduo que vem se desapontando socialmente (PONTES, 1998b).

Dos 237 casos de tentativa de suicídio analisados em 2003, ocorreram $2(0,84 \%)$ mortes e $219(92,41 \%)$ curas. O número de curas dos casos analisados no ano de 2004 foi de 279 (77,29\%), ocorrendo 9 (2,49\%) mortes. A proporção de casos em que não se confirmou cura e os casos ignorados foram, respectivamente, 9,70\% e 10,25\% sendo maior do que no ano de 2003 (5,96\% e 0,84\%) (Gráfico 3).

Baseado em estatísticas hospitalares, os casos não fatais de tentativa de suicídio são 50 a 100 vezes maiores do que os fatais (FISHER et al, 1993), confirmando o baixo número de óbitos relatados na pesquisa. 


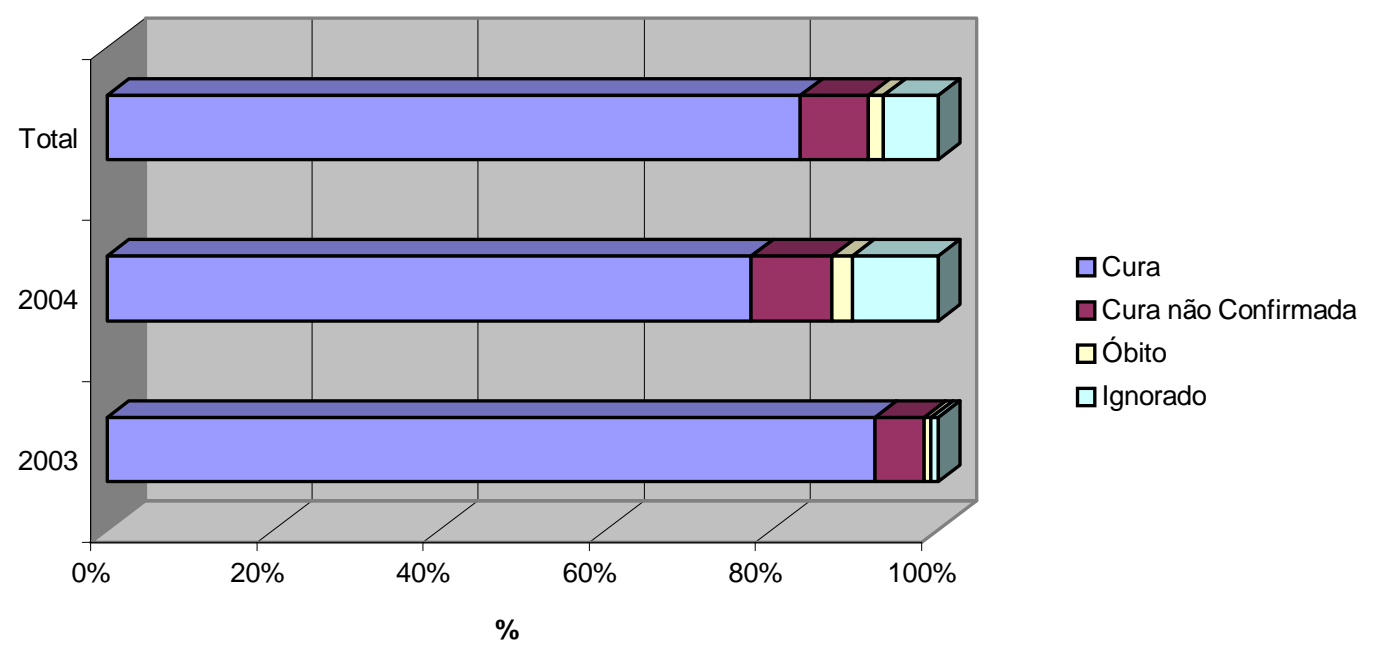

Gráfico 3: Evolução dos pacientes notificados pelo CIT-GO no ano de 2003 e 2004.

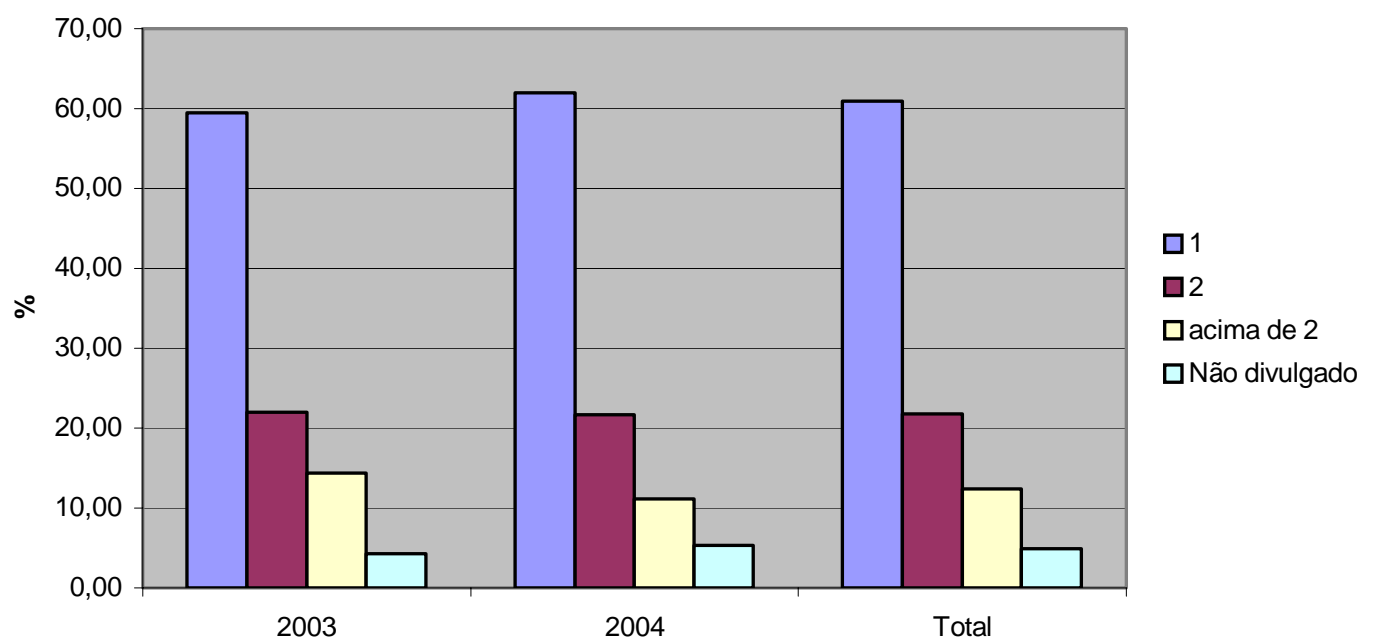

Gráfico 4: Quantidade de medicamentos associados e ingeridos por individuo intoxicado no período 2003-2004.

Em relação à associação medicamentosa, não existe diferença muito significativa entre os dois anos, de tal forma que $60,97 \%$ das tentativas de suicídio foram realizadas com um único medicamento. Uma fração de $21,78 \%$ dos pacientes se intoxicaram ingerindo 2 medicamentos e $12,40 \%$ com mais de 2 medicamentos, sendo que em $4,86 \%$ dos casos não foi informado a quantidade de medicamentos utilizada (Gráfico 4).

Dentre os medicamentos mais empregados nas tentativas de suicídio estão os benzodiazepínicos (aproximadamente 28\%) (Gráfico 5). Somente o diazepam foi responsável por aproximadamente 13\% do total de medicamentos ingeridos na tentativa de suicídio nesses anos. Os benzodiazepínicos, antigripais, antidepressivos, anti-inflamatórios são as classes de medicamentos que mais causam intoxicações em nosso país (BORTOLETTO \& BOCHNER, 1999). 


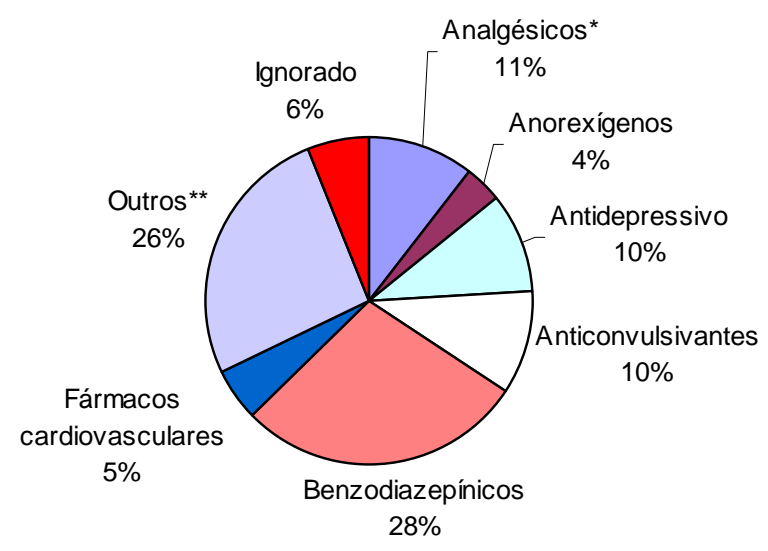

\section{A}

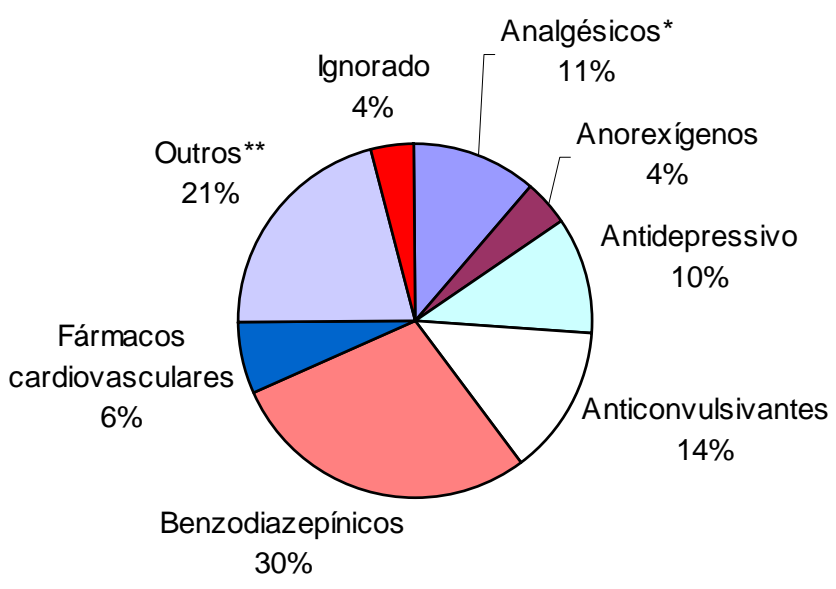

Gráfico 5: Principais classes de medicamentos usados para tentativa de suicídio notificados pelo CIT-GO nos anos de 2003 (Gráfico 5 A) e 2004 (Gráfico 5 B). Analgésicos representam os fármacos analgésicos, antipiréticos e antiinflamatórios

Os benzodiazepínicos (diazepam, clonazepam, bromazepam) são substâncias largamente usadas em todo o mundo. No Brasil, são prescritos por médico de cuidados primários e por diversas especialidades, sendo só a metade das prescrições dirigidas ao tratamento de transtornos psiquiátricos. A outra metade é dirigida ao tratamento de problemas diversos, como cardiovasculares, gastrointestinais, e músculo-esqueléticos, muitas vezes sem critérios bem definidos. Este relativo abuso nas prescrições, sem dúvida influencia na disponibilidade da droga no meio doméstico e, conseqüentemente, propicia o seu uso indevido (HARDMAN \& LIMBIRD et al, 2003; ANDRADE FILHO, 2001).

Os anticonvulsivantes foram o segundo grupo de maior incidência nas tentativas de auto-extermínio em 2004 e o terceiro em 2003. Neste período, só a incidência da carbamazepina foi de 6,28\% em 2003 e 10,47\% em 2004.

O terceiro grupo de maior incidência em 2004 e o segundo grupo em 2003 de maior incidência foi o grupo dos analgésicos, antipiréticos e antiinflamatórios estes representam $11 \%$ dos casos, sendo que o diclofenaco e a dipirona juntos representaram $58 \%$ dos casos de tentativa de suicídio no ano de 2004 . Já no ano de 2003 , o paracetamol representou $29 \%$ dos casos.

Diferentemente de recente estudo desenvolvido por KAPUR et al, 2005, na Inglaterra, onde os analgésicos aparecem em primeiro lugar nas tentativas de suicídio, contribuindo com mais de $50 \%$ da casuística; os 
psicofármacos ficaram em segundo lugar. Tal situação pode ser devida aos padrões de prescrição e de comercialização das especialidades envolvidas, da facilidade ou dificuldade em obtê-las.

A classe dos antidepressivos respondeu por aproximadamente 10\% dos casos de intoxicações. Em 2003 a amitriptilina (51\%) e a fluoxetina (31\%) foram responsáveis por $82 \%$ das intoxicações por antidepressivos e em 2004 por $93,5 \%$ (68\% e 25,50\% respectivamente). A intoxicação por antidepressivos tricíclicos (amtriptilina) é freqüente e potencialmente grave. No Brasil, situa-se entre os três primeiros grupos de medicamentos que levam a intoxicação aguda (muito relacionada à tentativa de auto-extermínio) (ANDRADE FILHO, 2001).

Os fármacos cardiovasculares foram responsáveis por $5 \%$ e $6 \%$ dos casos em 2003 e 2004 respectivamente, e os anorexígenos foram responsáveis por $4 \%$ dos casos no mesmo período.

Desta forma, existe uma clara predileção pelos fármacos psicotrópicos e os analgésicos e antiinflamatórios não-esteroidais nas tentativas de suicídio. Tais fármacos foram responsáveis, neste período de 2 anos, por mais de $60 \%$ de todos os casos de tentativas de suicídio registrados no Centro de Informação Tóxico-Farmacológicas de Goiás (CIT). Provavelmente, esta predominância deva-se, proporcionalmente, à distribuição de consumo de medicamentos no país, bem como ao conhecimento da eficácia dos mesmos, por provocarem "efeitos imediatos", principalmente relacionados à indução do sono e à diminuição das funções vitais (LEFĖVRE, 1987; SIMÕES \& FARACHE FILHO, 1988; ROSEMBERG, 1994).

Apesar da baixa letalidade, a morbidade é elevada, o que vem reacender a necessidade de vigilância, orientação e educação permanentes de profissionais de saúde e pacientes do sistema de saúde brasileiro para modificar estas mórbidas estatísticas envolvendo produtos medicamentosos, especialmente desenvolvidos para a promoção da saúde e que, no sentido literal e simbólico, podem também abreviar a vida, trazendo alento à frase de Paracelsus, que diz que "todo medicamento é veneno; é a dose que diferencia o veneno do remédio" (LEFÈVRE, 1987; LEFÈVRE, 1991).

Levantamentos estatísticos descritivos, da natureza deste realizado auxiliam-nos, também, a enfocar o ensino e a prática da Toxicologia para os grupos de produtos medicametosos realmente mais importantes epidemiologicamente, aumentando a eficiência nos diagnósticos e tratamentos de casos de intoxicações agudas, como nos grupos de fármacos supracitados. Adicionalmente, pode-se ampliar a capacidade de prevenção de casos de tentativas de suicídio, por meio do estudo das características psiquiátricas prevalentes daqueles que experimentam o auto-extermínio.

\section{AGRADECIMENTOS}

Ao Centro de Informações Tóxico-Farmacológicas do Estado de Goiás (CIT-GO) pela abertura do banco de dados de intoxicações em Goiás.

\section{REFERÊNCIAS BIBLIOGRÁFICAS}

AMDUR, M.O., DOULL, J. and KLAASSEN, C.D., Casarett and Doull's Toxicology, $4^{\text {th }}$ ed., 1033p., Pergamon Press, New York-NY, USA, 1991.

ANDRADE FILHO, A.; CAMPOLINA, D.; DIAS, M.B. Toxicologia na prática clínica. Belo Horizonte: Folium, 2001.

ANVISA: www.anvisa.gov.br (Guias de Urgência Biblioteca Virtual em Saúde/ANVISA), 2004.

BORTOLETTO, M. E; BOCHNER, R. Impacto dos medicamentos nas intoxicações humanas no Brasil Cad. Saúde Pública, Rio de Janeiro, v. 15, n. 4, p. 859-869, 1999.

ELLENHORN, M.J., Ellenhorn's Medical Toxicology - Diagnosis and Treatment of Human Poisoning, 2nd ed., Williams \& Wilkins, Baltimore-EUA, 1997.

FISHER A. J., ZIERVOGEL, C. F., CHALTON, D. O., LEGER, P. H., \& ROBERTSON, B. A.. Risk-taking behaviour of Cape Peninsula high-school students: Part II. Suicidal behaviour. South African Medical Journal, v. 83, p. 474476, 1993.

GOSSEL, T.A. \& BRICKER, J.D. Principles of Clinical Toxicology, 3rd edition, 447 p., Raven Press, New York, USA, 1994. 
HARDMAN, J.G. \& LIMBIRD, L.E. Goodman \& Gilman's The Phamacological Basis of Therapeutics, $10^{\text {th }}$ ed., McGraw Hill, New York-USA, 2003.

KAPUR, N., TURNBULL, P., HAWTON, K., SIMKIN, S., SUTTON, L., MACKWAY-JONES, K., BENNEWITH, O., GUNNELL, D. Self-poisoning suicides in England: a multicentre study. Q J Med, p. 1-9, jun 2005.

KOROLKOVAS, A.; CUNHA, B.C.A.; ALBUQUERQUE, F.F; FRANÇA, C. Dicionário Terapêutico Guanabara: Guanabara Koogan, 1999/2000.

LEFÈVRE, F. A oferta e a procura de saúde através do medicamento: proposta de um campo de pesquisa. Rev. Saúde Pública, v.21, n.1, p.64-67, 1987.

LEFÈVRE, F. O medicamento como mercadoria simbólica. São Paulo, Cortez, 1991.

MÍDIO A. F., Glossário de Toxicologia, Ed. Rocca, p:95, São Paulo, 1992

OGA, S., Fundamentos de Toxicologia, Ed. Atheneu, São Paulo-SP, 2004.

PONTES, C.P. Psiquiatria: conceitos e práticas. São Paulo (2ºd): Lemos Editorial, 1998a.

PONTES, C.P. Psiquiatria: conceitos e práticas. São Paulo ( $2^{\circ}$ ed). In PLATÃO. Diálogos III- A República. Tradução de Leonel Vallandro. Rio de Janeiro: Ediouro, Lemos Editorial, 1998b.

ROZEMBERG, B. O consumo de calmantes e o "problema de nervos" entre lavradores. Rev. Saúde Pública, v. 28, n...4, p.300-308, 1994.

SILVA, P. Farmacologia. Rio de Janeiro: Guanabara Koogan, 2002.

SIMÕES, M.J.S.; FARACHE FILHO, A. Consumo de medicamentos em região do Estado de São Paulo (Brasil), 1985. Rev. Saúde Pública, v. 22, n..6, São Paulo, 1988.

SINITOX - Sistema Nacional de Informações Tóxico-Farmacológicas (www.fiocruz.br/sinitox).

STENGEL, E. Suicide and attempted suicide. London: Penguin Books, 1964.

WHO - IPCS-INTOX: www.who.org/ipcs intox (International Program for Chemical Safety), 2005.

WHO - World Health Organization. The World Health Report 2001. Mental health: New understanding, new hope, 2001. 